\title{
Fréquence et comparaison des performances pour les génotypes à quatre loci affectant des protéines de l'œuf chez la poule
}

\author{
L. DURAND et P. MERAT \\ I.N.R.A., Laboratoire de Génétique factorielle \\ C.H.R.Z., 78350 Jouy-en-Josas
}

Résumé

Dans deux populations expérimentales aviaires, de 1973 à 1980, le génotype des poules en ponte a été identifié pour les 4 loci, $\mathrm{Ov}, \mathrm{G}_{2}, \mathrm{G}_{3}$ et $\mathrm{Tf}$, responsables de variants génétiques de la migration électrophorétique de protéines du blanc d'œuf. Deux allèles, A et B, étaient présents dans les deux populations aux loci $G_{2}$ et $G_{3}$, et dans l'une d'elles pour les loci $\mathrm{Ov}$ et Tf. Les fréquences alléliques observées indiquent une prépondérance de l'allèle A aux loci $\mathrm{Ov}$ et $\mathrm{G}_{3}$, et de l'allèle $B$ aux loci $\mathrm{G}_{2}$ et $\mathrm{Tf}$, en accord avec les observations de divers auteurs. Nous retrouvons un linkage, signalé antérieurement, entre les loci Ov et $\mathrm{G}_{3}$, avec une fréquence de recombinaison estimée d'environ 7 p. 100.

La comparaison des performances de croissance et de ponte, et des caractéristiques des œufs entre génotypes à ces quatre loci sur des couples de sœurs ou demi-sœurs, ne fait apparaître, dans l'ensemble, qu'un petit nombre de différences significatives; la réalité de celles-ci serait à confirmer par des données complémentaires. Pour le taux d'éclosion, la seule comparaison statistique possible en effectifs suffisants, sur des mères de différents génotypes accouplées aux mêmes pères, faisait ressortir une différence significative, relative au locus $G_{3}$. D'autre part, l'examen des proportions mendéliennes dans les descendances par type de croisement relatif à chaque locus, faisait apparaître certains écarts très hautement significatifs par rapport à la prévision théorique. L'explication de ces écarts par des différences de mortalité embryonnaire ou post-embryonnaire de certains génotypes, n'est pas évidente dans les données présentes.

\section{I. - Introduction}

On connaît depuis les années 1960 (LuSh, 1961; BAKer \& MANwell, 1962 ; OGDen et al., 1962) l'existence de variants génétiques de plusieurs protéines du blanc d'œuf chez la poule. Depuis, de nombreux travaux ont été publiés à leur sujet, et il n'est pas question de les citer exhaustivement. Quelques ouvrages généraux ont fait le point sur les connaissances relatives à ces variants, tels BAKER (1968 a), BAKER (1968 b), BAKER et al. (1970), Gilbert (1971). 
Quatre loci présentent un polymorphisme fréquent dans des populations aviaires : Ov (ovalbumine), $\mathrm{G}_{2}, \mathrm{G}_{3}$ (ovoglobulines), Tf (conalbumines). De nombreux auteurs, dont certains cités dans les ouvrages précédents, ont cherché des associations éventuelles du génotype à ces loci avec des caractères de production ou relatifs à la survie embryonnaire ou post-embryonnaire. Au total, les résultats publiés paraissent peu concluants. Nous avons cependant voulu observer, dans notre troupeau expérimental où ces mêmes polymorphismes étaient identifiés, les performances des génotypes à chaque locus pour diverses caractéristiques de production et de viabilité.

\section{II. - Matériel et méthodes}

Les données recueillies portent sur le troupeau pedigree expérimental du laboratoire, de 1973 à 1980 inclus. Dans ce troupeau reproduit annuellement, les reproducteurs étaient choisi de façon que toutes les familles d'effectif suffisant soient représentées. En outre, certains gènes à effet visible étaient maintenus en ségrégation, et une faible intensité de sélection était exercée sur les performances de ponte et de reproduction. Par contre, aucun choix conscient n'était fait sur les génotypes responsables des protéines de l'œuf. Ce troupeau était issu à l'origine, en 1954-1955, d'un croisement entre plusieurs races, incluant la Rhode-Island rouge, la Wyandotte, la Gâtinaise, la Bresse et la Marans, comme indiqué dans des articles précédents (Mérat, 1967, 1970). Il contenait deux sous populations d'origine en partie commune, l'une (population 1) éclose au printemps de chaque année, l'autre (population 2) en automne. Les poules de la population 1 étaient de taille normale (allèle $\mathrm{Dw}$ ), celles de la population 2 possédaient le gène dw de «nanisme» lié au sexe, apparu à l'origine dans la première (MéraT, 1969). Ces deux populations, reproduites toutes deux en troupeau fermé, se distinguent l'une de l'autre par la fréquence des génotypes aux loci étudiés. Par contre, pour les proportions des ségrégations mendéliennes intra-descendances et pour les comparaisons des performances entre génotypes, nous les avons regroupées, car elles ne présentaient pas d'hétérogénéité décelable de ce point de vue.

La reproduction était faite par accouplements naturels en parquets pedigree. Les jeunes étaient élevés au sol, puis les poulettes étaient mises en cages individuelles à partir de l'âge de 16 semaines. La ponte était enregistrée jusqu'à l'âge approximatif de 10 mois. Le poids et diverses caractéristiques des œufs étaient notés pendant 2 semaines avant la fin du contrôle de ponte. Les oiseaux étaient pesés individuellement aux âges de 8 semaines et 10 mois. La mortalité était, dans l'ensemble, faible et n'a pas été prise en considération dans le présent travail. Le tableau 1 précise la définition des mesures prises sur chaque individu.

Le pourcentage de poussins éclos, rapporté d'une part au nombre d'œufs incubés, de l'autre au nombre d'œufs fécondés, était d'autre part relevé pour chaque reproducteur, pour la période d'éclosion pedigree qui s'étendait sur 5 ou 6 semaines. Les œufs «non fécondés » ou «clairs » étaient identifiés par simple mirage; il est donc possible qu'ils contiennent une certaine proportion d'embryons à mortalité très précoce. 


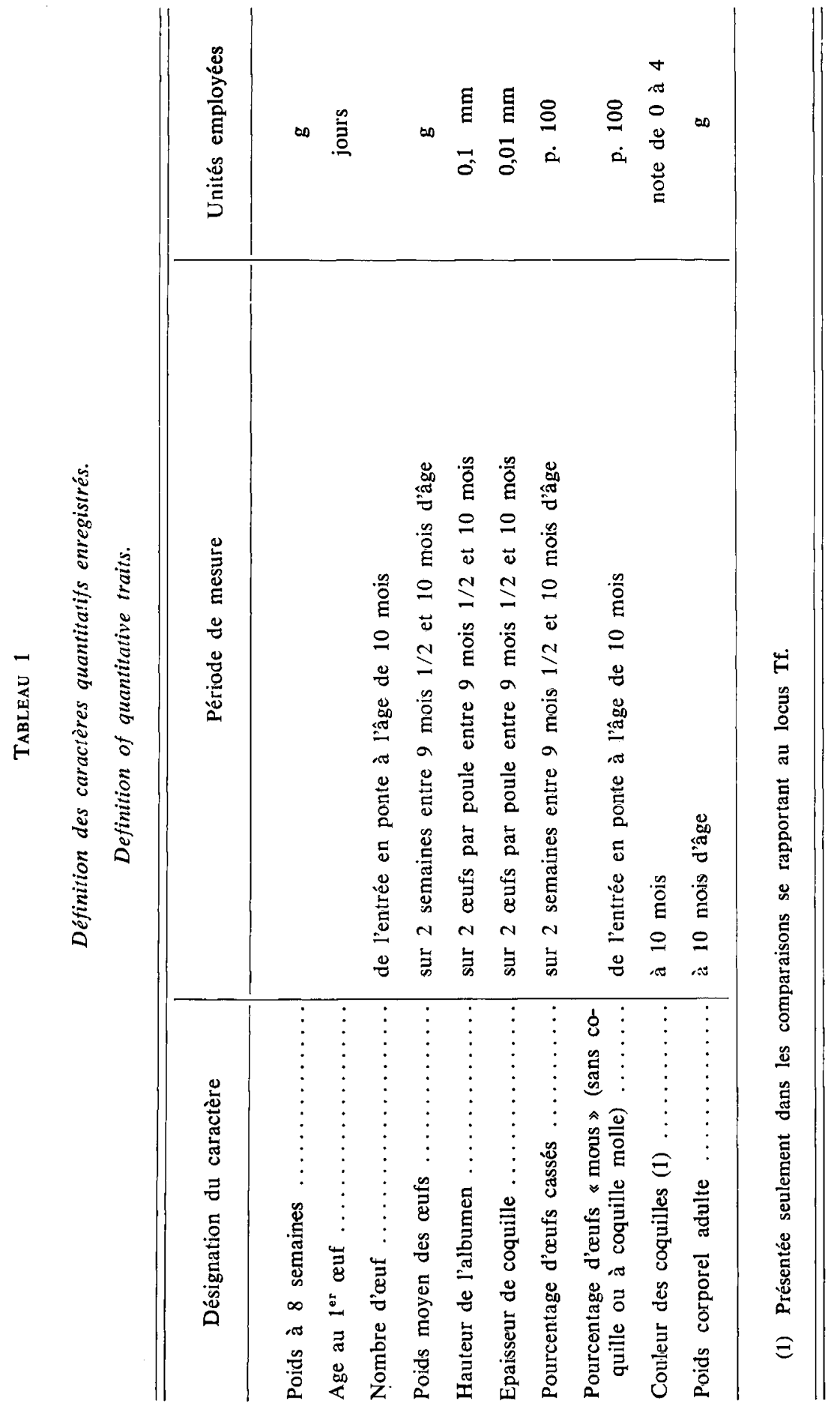


Quatre loci responsables d'une modification de la migration électrophorétique d'une protéine de l'albumen de l'œuf étaient étudiés ici : Ov (ovalbumine), $\mathrm{G}_{2}, \mathrm{G}_{3}$ (ovoglobulines), Tf (conalbumines). L'identification des génotypes pour chacun était faite par électrophorèse sur gel d'amidon à une concentration de 11 p. 100 , sur l'albumen d'un œuf par poule, prélevé sur la ponte d'une semaine entre les âges de 9 et 10 mois.

Le système de tampons utilisé était celui de GAHNE (1966); pour les cuves : tampon acide borique-hydroxyde de lithium $(\mathrm{pH}=8,5)$; pour le gel : tampon tris-citrate $(\mathrm{pH}=8,6)$ mélangé dans les proportions 5,5:1 avec le tampon cuve. La migration se poursuivait pendant 5 heures sous une tension de 8 volts $/ \mathrm{cm}$. Les protéines étaient révélées par le noir amide.

Dans la population 1, les mêmes génotypes que ceux trouvés dans la présente étude avaient déjà été identifiés plusieurs années auparavant par CRolziER (1969).

\section{Analyse statistique des résultats}

Les fréquences des génotypes sont calculées par locus ; leur comparaison à celles prévisibles par panmixie n'est pas indiquée étant donnés les écarts possibles à la panmixie discutés plus loin. Par ailleurs, pour chaque type d'accouplement relatif au génotype à chaque locus, les proportions observées dans la descendance femelle sont comparées par un test de $\chi^{2}$ aux proportions mendéliennes prévues.

Pour les caractères quantitatifs définis au tableau 1, les génotypes à chaque locus sont comparés deux à deux, sur des couples de sœurs ou demi-sœurs de même père, par un test $t$. La méthode des couples permet de cumuler pour un caractère donné les différences entre membres d'un couple et d'obtenir un test unique sur l'ensemble des années. Quant au taux d'éclosion, seuls les pères auxquels étaient accouplées des mères de génotype différent pour le locus étudié étaient pris en considération pour un test statistique. Les pourcentages d'éclosion (sur œufs incubés ou sur œufs fécondés) pour deux génotypes distincts de mères appariées à un père donné constituaient un couple, et ces couples étaient totalisés pour les différents pères et comparés par un test $\mathrm{t}$.

\section{III. - Résultats}

\section{Génotypes identifiés et fréquences}

Le tableau 2 indique, par locus et par sous-population (1 ou 2), les génotypes présents et leurs fréquences, ainsi que les fréquences des allèles $\mathbf{A}$.

\section{Proportions mendéliennes}

Le tableau 3 présente les propositions mendéliennes, sur le total des années pour chaque type d'accouplement à chaque locus. 


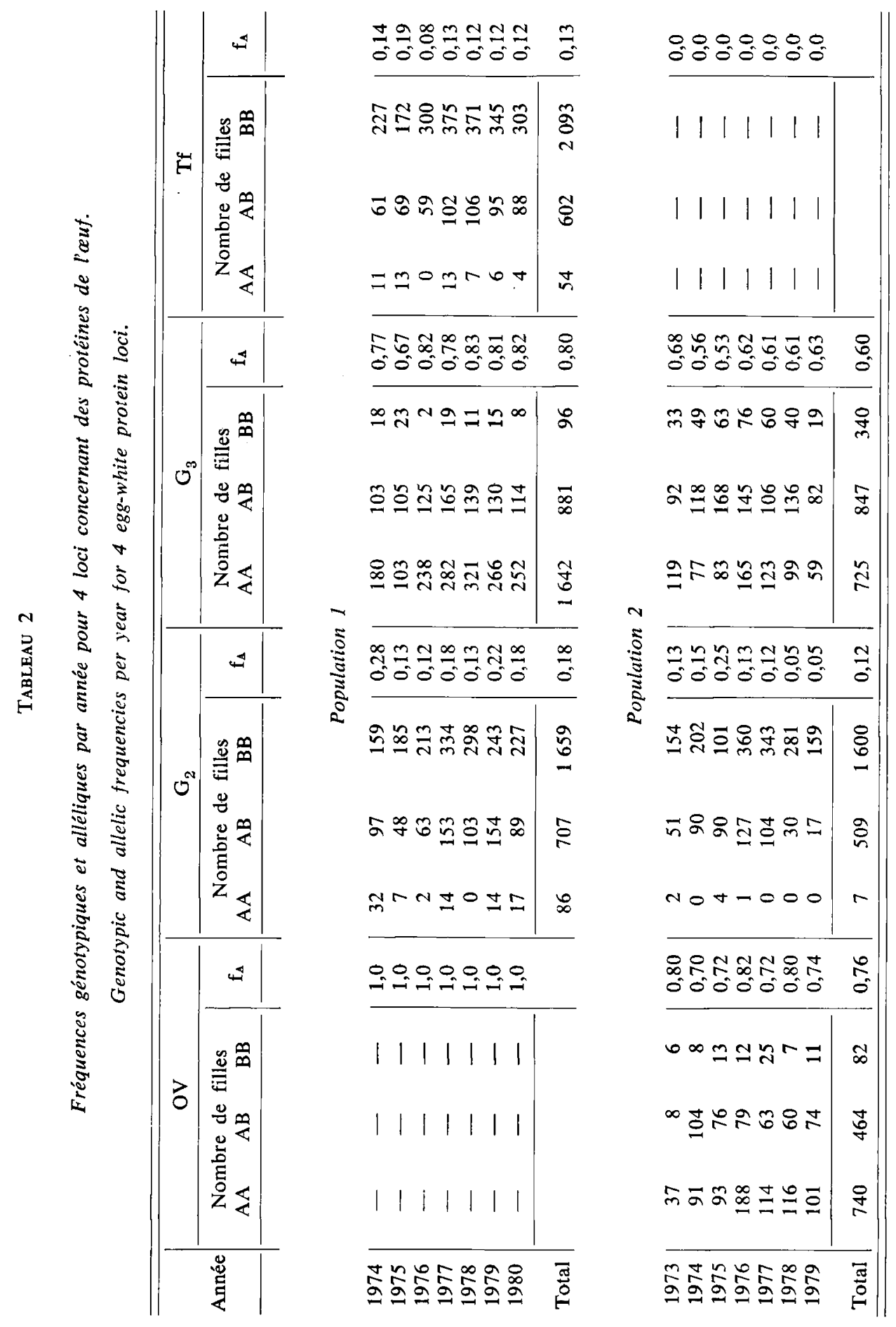




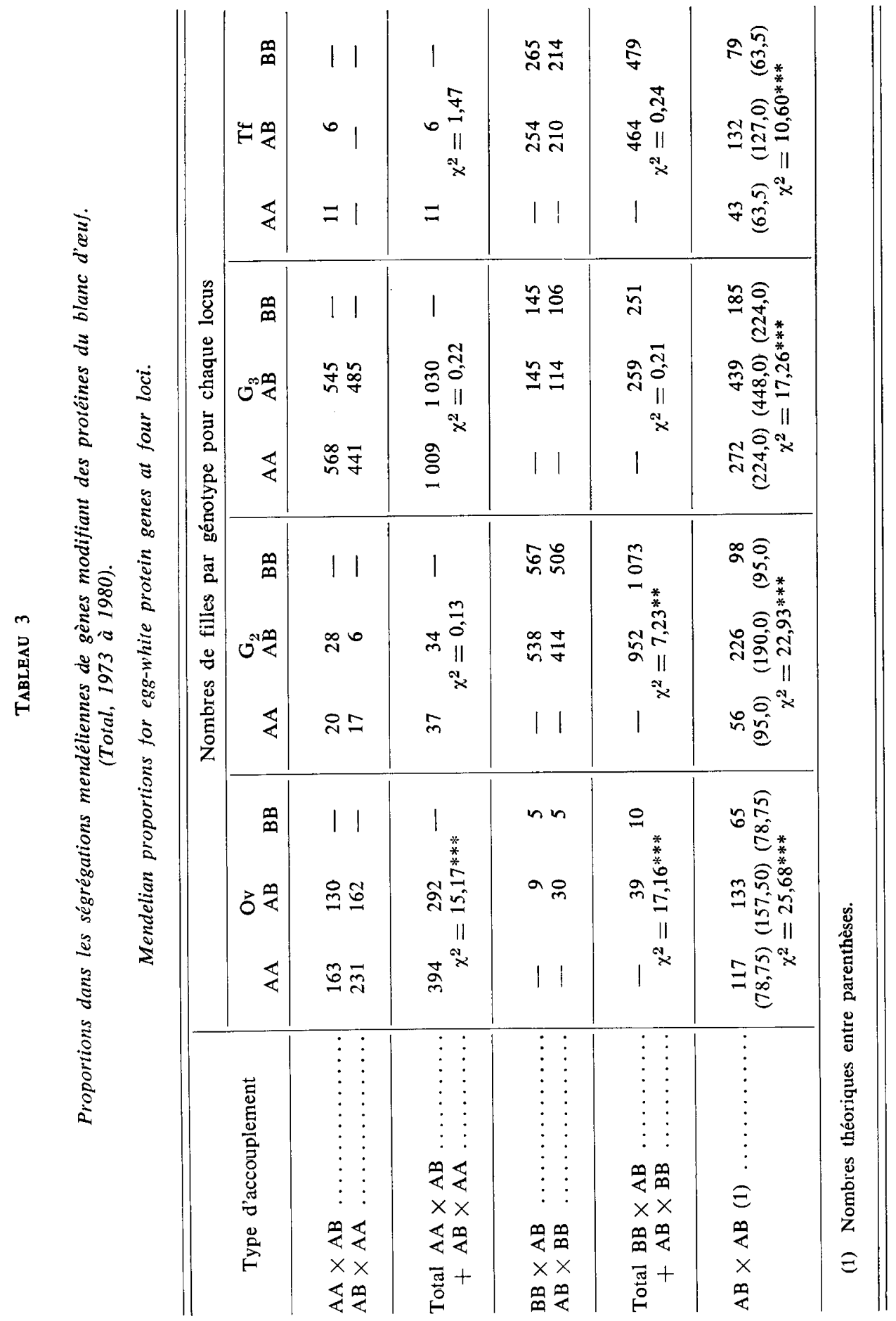




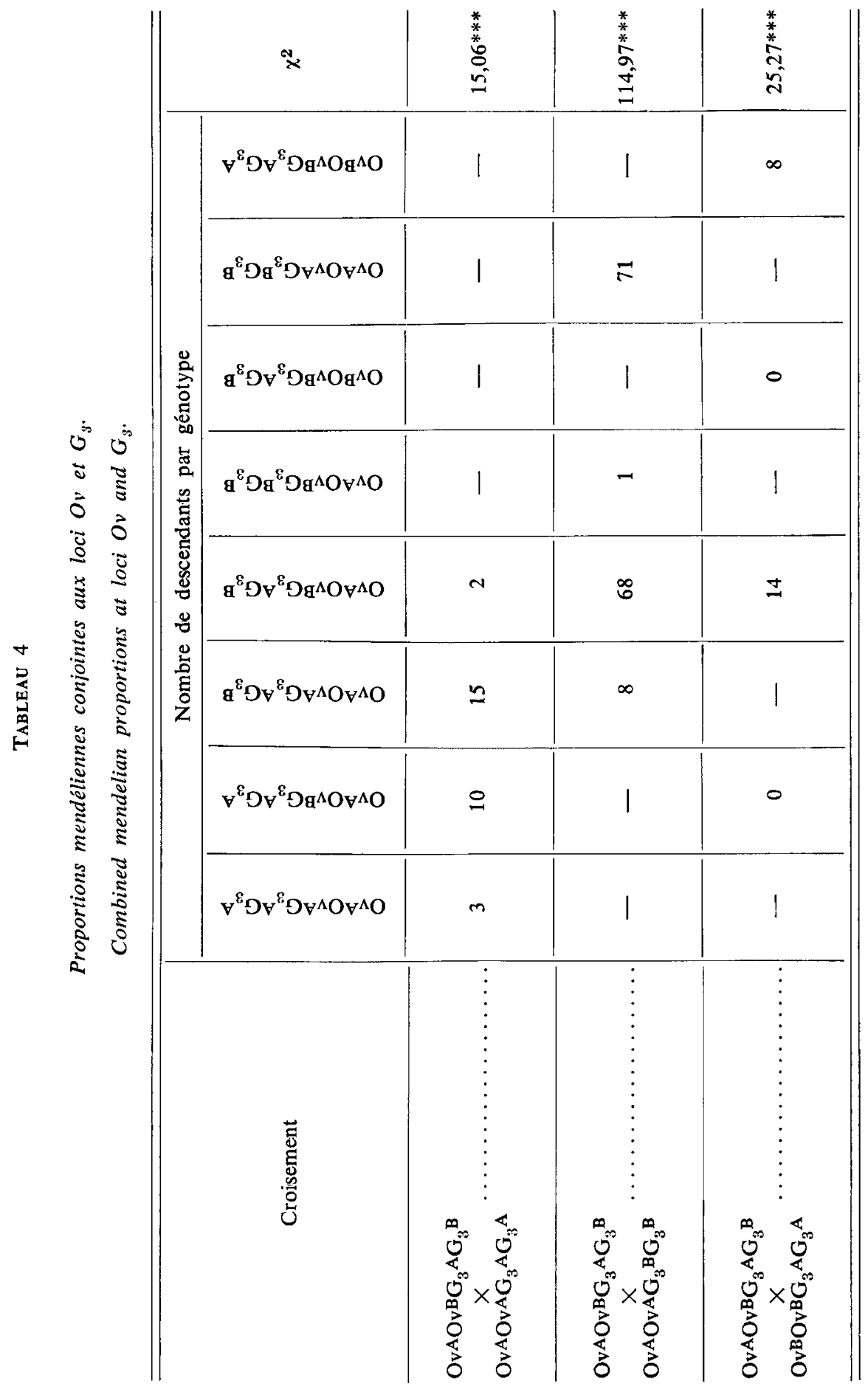




\section{Linkage $O v-G_{3}$}

$\mathrm{Au}$ tableau 4 figurent les proportions mendéliennes conjointes aux loci $\mathrm{Ov}$ et $\mathrm{G}_{3}$, dans la descendance des parents dont l'un est double hétérozygote.

\section{TABleau 5}

Comparaison des performances pour les génotypes $A A$ et $A B$ au locus $O v$ : total des couples, 1973 à 1979 (population 2 : naines).

Comparison of performance traits for $A A$ and $A B$ genotypes at $O v$ locus : total of pairs from 1973 to 1979 (population 2 : dwarfs).

\begin{tabular}{|c|c|c|c|c|}
\hline \multirow{2}{*}{ Caractères } & \multirow{2}{*}{$\mathrm{N}$} & \multicolumn{2}{|c|}{ Valeur moyenne } & \multirow{2}{*}{$\mathbf{t}$} \\
\hline & & AA & $\mathrm{AB}$ & \\
\hline Poids 8 s. (g) $\ldots \ldots \ldots \ldots \ldots \ldots \ldots$ & 128 & 548,2 & 551,4 & $-0,34$ \\
\hline Age au $1^{\text {er }}$ œuf $(j) \ldots \ldots \ldots \ldots$ & 150 & 158,2 & 156,6 & 1,50 \\
\hline $\begin{array}{l}\text { Nombre d'œufs (de l'entrée en ponte } \\
\text { à } 10 \text { mois d'âge) } \ldots \ldots \ldots \ldots \ldots \ldots \ldots\end{array}$ & 150 & 82,2 & 82,2 & 0,01 \\
\hline Poids moyen des œufs $(g) \ldots \ldots \ldots \ldots$ & 131 & 52,85 & 53,06 & $-0,46$ \\
\hline Hauteur albumen $(0,1 \mathrm{~mm}) \ldots \ldots$ & 133 & 67,0 & 65,5 & 1,43 \\
\hline Epaisseur coquille $(0,01 \mathrm{~mm})$ & 133 & 36,1 & 36,6 & $-1,39$ \\
\hline Cufs cassés' (p. 100) & 88 & 3,75 & 4,69 & $-0,69$ \\
\hline Eufs mous (p. 100) & 128 & 1,42 & 0,58 & $2,79 * *$ \\
\hline Poids adulte $(\mathrm{g}) \ldots$ & 149 & 1806 & 1811 & $-0,15$ \\
\hline
\end{tabular}

\section{Comparaison des performances}

Les tableaux 5, 6, 7 et 8 indiquent les performances autres que le taux d'éclosion totalisées sur les couples de sœurs ou demi-sœurs pour l'ensemble des années, pour les génotypes représentés par des effectifs suffisants, respectivement aux loci Ov, $\mathrm{G}_{2}$, $\mathrm{G}_{3}$ et $\mathrm{Tf}$.

Le tableau 9 contient le taux d'éclosion (poussins nés / œufs incubés et poussins nés / œufs fécondés) pour les génotypes de mères obtenus en nombre suffisant aux différents loci. Enfin, à titre indicatif, le tableau 10 donne, pour toutes les années groupées et par locus, les taux d'éclosion moyens pour tous les génotypes paternels et maternels combinés. 
TABlEau 6

Génotypes au locus $G_{2}$ et performances (total 1973-1980).

Performance traits according to genoiype at $G_{2}$ locus (total 1973-1980).

\begin{tabular}{|c|c|c|c|c|c|c|c|c|}
\hline \multirow{2}{*}{$\begin{array}{c}\text { Génotypes } \\
\text { comparés } \\
\text { et caractères }\end{array}$} & \multicolumn{4}{|c|}{ Population 1 (normales) } & \multicolumn{4}{|c|}{ Population 2 (naines) } \\
\hline & $\mathrm{N}$ & $\bar{x}_{1}(*)$ & $\overline{\mathrm{x}} 2$ & $\mathrm{t}$ & $\mathrm{N}$ & $\overline{\mathrm{x}}_{1}$ & $\overline{\mathrm{x}}_{2}$ & $\mathrm{t}$ \\
\hline 1. $A A$ et $A B$ & & & & & & & & \\
\hline Poids 8 s. $\ldots \ldots \ldots$ & 48 & 797,8 & 807,9 & $-0,60$ & & & & \\
\hline Age au $1^{\text {er }}$ œuf $\ldots$ & 76 & 138,0 & 141,0 & $-1,66$ & & & & \\
\hline Nombre d'œufs .... & 75 & 104,2 & 97,7 & $2,37 *$ & & & & \\
\hline Poids moyen des œufs & 68 & 51,43 & 51,98 & $-1,46$ & & & & \\
\hline Hauteur albumen ... & 73 & 59,2 & 59,7 & $-0,36$ & & & & \\
\hline Epaisseur coquille .. & 71 & 37,0 & 37,3 & $-0,79$ & & & & \\
\hline Eufs cassés (p. 100) & 13 & 4,15 & 1,53 & 0,89 & & & & \\
\hline Eufs mous (p. 100) & 11 & 2,16 & 1,46 & 0,99 & & & & \\
\hline Poids adulte $\ldots \ldots$ & 54 & 2450 & 2480 & $-0,51$ & & & & \\
\hline 2. $A B$ et $B B$ & & & & & & & & \\
\hline Poids 8 s. $\ldots \ldots \ldots$ & 331 & 744,0 & 745,4 & $-0,09$ & 85 & 574,5 & 577,1 & $-0,26$ \\
\hline Age au $1^{\text {er }}$ œuf $\ldots$ & 417 & 146,6 & 145,1 & 1,81 & 74 & 155,5 & 156,0 & $-0,33$ \\
\hline Nombre d'œufs .... & 417 & 95,6 & 97,1 & $-1,48$ & 106 & 82,9 & 82,2 & 0,33 \\
\hline Poids moyen des œufs & 390 & 53,54 & 53,63 & $-0,33$ & 97 & 53,62 & 53,06 & 1,16 \\
\hline Hauteur albumen... & 384 & 59,5 & 59,4 & 0,11 & 98 & 68,5 & 67,9 & 0,56 \\
\hline Epaisseur coquille .. & 384 & 36,8 & 36,7 & 0,46 & 99 & 37,1 & 36,6 & 1,10 \\
\hline Eufs cassés (p. 100) & 206 & 5,87 & 4,87 & $-0,25$ & 56 & 4,98 & 5,02 & $-0,02$ \\
\hline CEufs mous (p. 100) & 112 & 2,65 & 2,49 & 0,22 & 24 & 0,30 & 1,80 & $-1,65$ \\
\hline Poids adulte...$\cdots$ & 410 & 2402 & 2433 & $-1,32$ & 105 & 1835 & 1859 & $-0,74$ \\
\hline
\end{tabular}

$\left(^{*}\right) \bar{x}_{1}$ et $\overline{\mathbf{x}}_{2}$ désignent respectivement, dans l'ordre, les valeurs moyennes des deux génotypes indiqués dans la première colonne. 


\section{TABlEaU 7}

Génotypes au locus $G_{3}$ et performances (total 1973-1980).

Performance traits according to genotype at $G_{3}$ locus (total 1973-1980).

\begin{tabular}{|c|c|c|c|c|c|c|c|c|}
\hline \multirow{2}{*}{$\begin{array}{c}\text { Génotypes } \\
\text { comparés } \\
\text { et caractères }\end{array}$} & \multicolumn{4}{|c|}{ Fopulation 1 (normales) } & \multicolumn{4}{|c|}{ Population 2 (naines) } \\
\hline & $\mathrm{N}$ & $\bar{x}_{1}\left(^{*}\right)$ & $\overline{\mathbf{x}}_{2}$ & $\mathrm{t}$ & $\mathrm{N}$ & $\overline{\mathbf{x}}_{1}$ & $\overline{\mathrm{x}}_{2}$ & $\mathrm{t}$ \\
\hline \multicolumn{9}{|l|}{ 1. $A A$ et $A B$} \\
\hline Poids $8 \mathrm{~s}$. & 363 & 740.7 & 745,7 & 0,74 & 96 & 548,2 & 554,1 & $-0,58$ \\
\hline Age au $1^{\text {er }}$ ouf & 507 & 148,2 & 147,8 & 0,14 & 97 & 155,4 & 156,9 & $-1,06$ \\
\hline Nombre d'œufs & 514 & 94,4 & 96,4 & 1,68 & 136 & 94,7 & 91,8 & 1,58 \\
\hline Poids moyen des œufs & 478 & 53,39 & 53,39 & 0,03 & 112 & 53,18 & 53,35 & $-0,35$ \\
\hline Hauteur albumen ... & 480 & 60,4 & 61,0 & $-1,21$ & 117 & 66,3 & 66,5 & $-0,13$ \\
\hline Epaisseur coquille .. & 466 & 37,0 & 36,9 & 0,61 & 118 & 36,8 & 37,0 & $-0,49$ \\
\hline CEufs cassés (p. 100) & 269 & 4,38 & 6,00 & $-2,17 *$ & 77 & 6,93 & 6,06 & 0,49 \\
\hline Eufs mous (p. 100) & 142 & 2,72 & 2,70 & 0,26 & 20 & 0,60 & 0,36 & 0,98 \\
\hline Poids adulte ...... & 432 & 2454 & 2454 & 0,00 & 123 & 1769 & 1784 & $-0,63$ \\
\hline \multicolumn{9}{|l|}{ 2. $A A$ et $B B$} \\
\hline Poids $8 \mathrm{~s}$. & 20 & 800,4 & 753,0 & $3,13 *$ & 25 & 573,9 & 559,1 & 1,04 \\
\hline Age au $1^{\text {er }}$ œuf & 38 & 142,8 & 147,3 & $-1,53$ & 25 & 156,9 & 155,6 & 0,49 \\
\hline Nombre d'œufs & 43 & 95,6 & 94,9 & 0,12 & 30 & 88,1 & 88,6 & $-0,22$ \\
\hline Poids moyen des oufs & 37 & 51,87 & 53,01 & $-1,65$ & 27 & 53,26 & 53,26 & 0,00 \\
\hline Hauteur albumen ... & 35 & 60,7 & 60,4 & 0,43 & 29 & 67,3 & 66,9 & 0,13 \\
\hline Epaisseur coquille & 35 & 35,3 & 36,1 & $-1,46$ & 29 & 36,8 & 36,0 & 0,73 \\
\hline Eufs cassés (p. 100) & 27 & 2,60 & 5,60 & 1,29 & 21 & 5,21 & 3,22 & 1,02 \\
\hline Cufs mous (p. 100) & 11 & 2,16 & 1,46 & 0,99 & 14 & 0,62 & 0,67 & $-0,12$ \\
\hline Poids adulte ...... & 36 & 2742 & 2610 & 1,69 & 30 & 1836 & 1816 & 0,38 \\
\hline \multicolumn{9}{|l|}{ 3. $A B$ et $B B$} \\
\hline Poids $8 \mathrm{~s}$. & 61 & 696,5 & 729,7 & $-2,39 *$ & 119 & 572,0 & 572,2 & $-0,10$ \\
\hline Age au $1^{\text {er }}$ œuf & 109 & 149,1 & 148,0 & 0,61 & 121 & 156,8 & 156,8 & $-0,06$ \\
\hline Nombre d'œufs & 114 & 95,4 & 94,9 & 0,19 & 137 & 86,1 & 84,5 & 0,78 \\
\hline Poids moyen des œufs & 101 & 51,78 & 53,02 & $-2,76^{*}$ & 118 & 54,18 & 53,69 & 1,12 \\
\hline Hauteur albumen ... & 100 & 60,5 & 62,3 & $-1,54$ & 118 & 66,0 & 66,3 & $-0,25$ \\
\hline Epaisseur coquille .. & 102 & 36,3 & 36,5 & $-0,57$ & 127 & 36,5 & 35,8 & 1,52 \\
\hline CEufs cassés (p. 100) & 33 & 2,87 & 5,33 & $-1,02$ & 91 & 3,51 & 3,52 & $-0,00$ \\
\hline Eufs mous (p. 100) & 25 & 2,08 & 2,07 & 0,00 & 41 & 0,69 & 1,63 & $-1,54$ \\
\hline Poids adulte ....... & 102 & 2393 & 2470 & $-0,59$ & 135 & 1934 & 1961 & $-0,91$ \\
\hline
\end{tabular}

(*) $\overline{\mathbf{x}}_{1}$ et $\overline{\mathbf{x}}_{2}$ désignent respectivement, dans l'ordre, les valeurs moyennes des deux génotypes indiqués dans la première colonne. 


\section{Tableau 8}

Génotype au locus Tf et performarces (total 1974 à 1980) (population 1). Performance traits according to genotype at Tf locus (to:al 1974 to 1980) (population 1).

\begin{tabular}{|c|c|c|c|c|}
\hline $\begin{array}{c}\text { Génotypes comparés } \\
\text { et caractères }\end{array}$ & $\mathrm{N}$ & $\left.\bar{x}_{1}{ }^{*}\right)$ & $\overline{\mathbf{x}}_{2}$ & $\mathrm{t}$ \\
\hline 1. $A A$ et $A B$ & & & & \\
\hline Poids 8 s. $\ldots \ldots \ldots \ldots$ & 19 & 754,2 & 787,8 & $-1,00$ \\
\hline Age $1^{\text {er }}$ ouf $\ldots \ldots$ & 35 & 143,1 & 139,4 & 1,57 \\
\hline Nombre d'œufs & 35 & 104,5 & 108,7 & $-0,98$ \\
\hline Poids moyen des cuîs. & 32 & 52,87 & 52,59 & 0,34 \\
\hline Hauteur albumen & 32 & 56,4 & 58,2 & $-0,86$ \\
\hline Epaisseur coquille & 32 & 36,3 & 35,8 & 0,68 \\
\hline CEufs cassés (p. 100) & 9 & 4,18 & 5,05 & $-0,24$ \\
\hline Eufs mous (p. 100) & 13 & 3,23 & 2,79 & 0,28 \\
\hline Poids adulte ..... & 28 & 2463 & 2506 & $-0,76$ \\
\hline Conleur coquille. & 32 & 3,96 & 4,15 & $-0,71$ \\
\hline 2. $A B$ et $B B$ & & & & \\
\hline Poids 8 s. $\ldots \ldots$ & 262 & 785,2 & 775,8 & 1,43 \\
\hline Age $1^{\text {er }}$ œuf $\ldots$ & 351 & $i 43,5$ & 144,1 & 0,66 \\
\hline Nombre d'œufs .. & 351 & 102,2 & 100,9 & 0,87 \\
\hline Poids moyen des œufs & 311 & 54,07 & 53,51 & $2,26^{*}$ \\
\hline Hauteur albumen .. & 310 & 59,6 & 59,9 & 0,37 \\
\hline Epaisseur coquille $\ldots \ldots$ & 312 & 36,6 & 36,4 & 1,25 \\
\hline Eufs cassés (p. 100) ... & 180 & 5,91 & 6,23 & 0,27 \\
\hline Eufs mous (p. 100) & 161 & 3,79 & 3,18 & 0,56 \\
\hline Poids adulte & 309 & 2542 & 2453 & $2,25 *$ \\
\hline Couleur coquille $\ldots$ & 311 & 4,36 & 4,13 & $2,64 * *$ \\
\hline
\end{tabular}

(*) $\bar{x}_{1}$ et $\tilde{\mathbf{x}}_{2}$ désignent respectivement, dans l'ordre, les valeurs moyennes des deux génotypes indiqués dans la première colonne. 


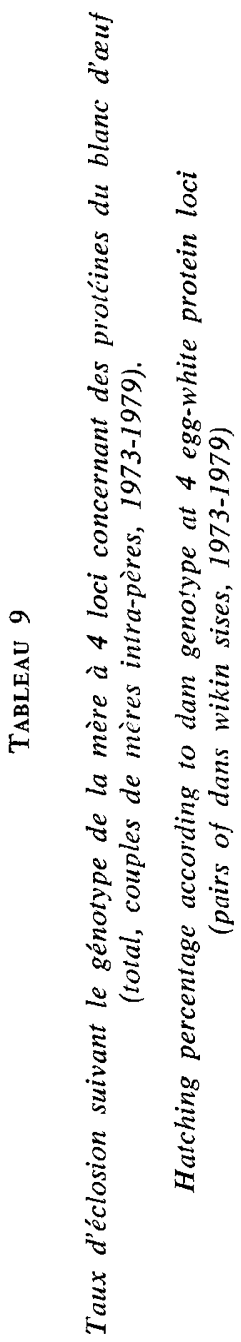

\begin{tabular}{|c|c|c|c|c|c|c|}
\hline \multirow{3}{*}{$\breve{H}$} & $\mid \hat{x}$ & & 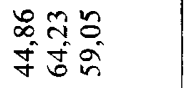 & & 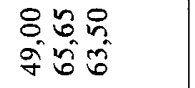 & \\
\hline & $\mid \vec{x}$ & & 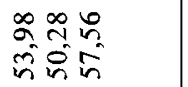 & & 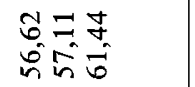 & \\
\hline & $\mathbf{z}$ & & $=\infty \bar{\sigma}$ & & $=\infty \bar{a}$ & $\stackrel{0}{0}$ \\
\hline \multirow{3}{*}{$0^{\circ}$} & $x^{*}$ & & 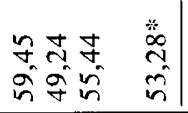 & & 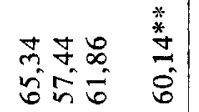 & 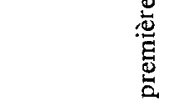 \\
\hline & $\mid \vec{x}$ & & 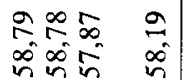 & & 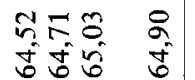 & 题 \\
\hline & $z$ & & œ & & జూ &. \\
\hline \multirow{3}{*}{ نी } & $x^{N}$ & & 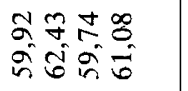 & & 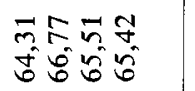 & 总 \\
\hline & $\mid \vec{x}$ & & 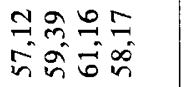 & & 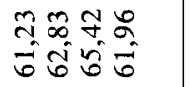 & $\tilde{v}$ \\
\hline & $z$ & & 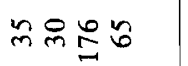 & & ติลี & 离 \\
\hline \multirow{3}{*}{ o } & $\mid x^{2}$ & & 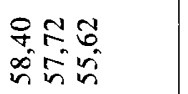 & & 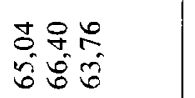 & $\underset{\frac{\pi}{\pi}}{\frac{\pi}{\pi}}$ \\
\hline & $x$ & & 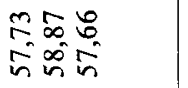 & & 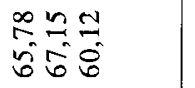 & \\
\hline & $z$ & & $n+n$ & & $8+0$ & 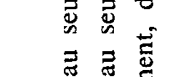 \\
\hline \multicolumn{2}{|c|}{ 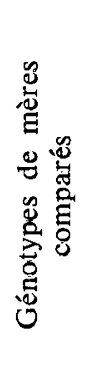 } & 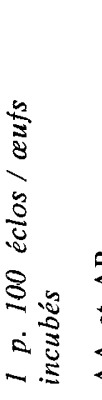 & 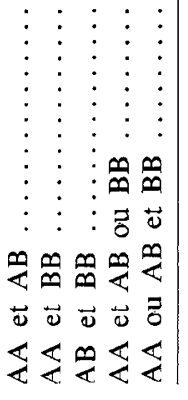 & 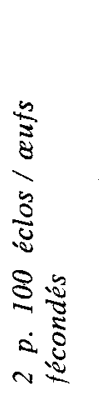 & 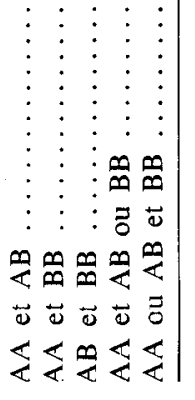 & 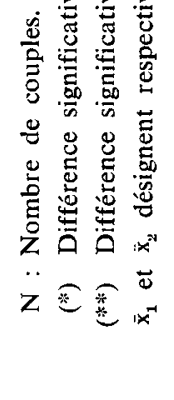 \\
\hline
\end{tabular}




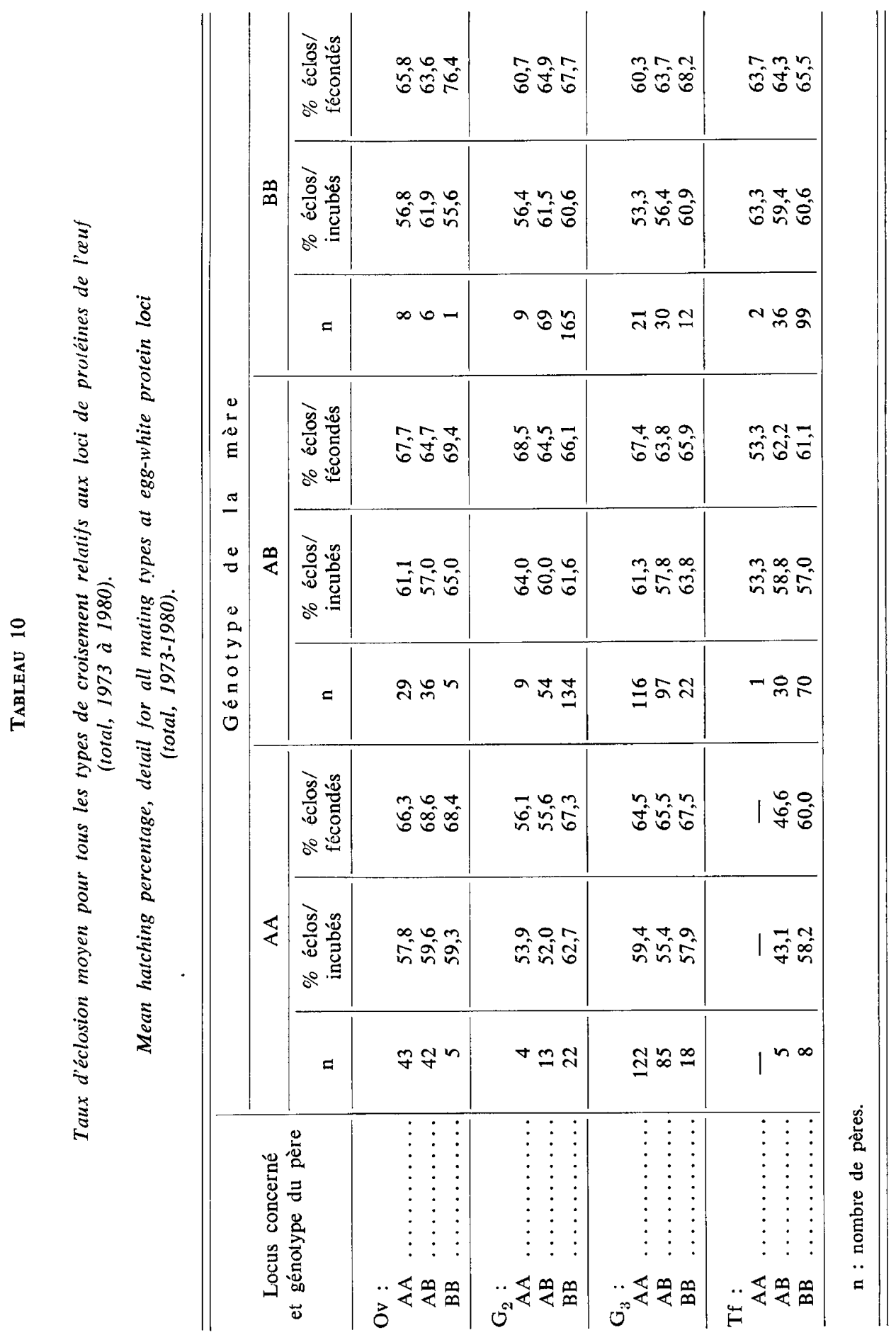




\section{IV. - Discussion et conclusions}

\section{Génotypes identifiés et fréquences; relations de linkage}

Les allèles rencontrés dans les deux populations et leurs fréquences paraissent conformes aux observations de la plupart des auteurs antérieurs (par exemple LuSH, 1961 ; Croizier, 1966 ; BaKer, 1968 a, 1968 b ; Manwell \& BaKer, 1970 ; Stratil, 1970) : prépondérance de l'allèle $A$ au locus $O v$ et au locus $G_{3}$, de l'allèle $B$ au locus $G_{2}$ et au locus Tf. L'une des deux populations est fixée pour OvA, l'autre pour $\mathrm{Tf}^{\mathrm{B}}$.

Les fréquences alléliques (tableau 2) ne montrent pas de tendance nette dans leurs fluctuations d'année en année. Ces dernières peuvent s'expliquer par l'échantillonnage des reproducteurs (une vingtaine de mâles par population pour chaque génération). Pour cette même raison, il nous a paru inutile de tester chaque année pour chaque locus la conformité des proportions génotypiques observées à celles attendues dans l'hypothèse de panmixie (loi de Hardy-WeINBERg). Sur le total des années, le $\chi^{2}$ testant cette conformité n'est, toutefois, significatif que dans deux cas, pour les loci $G_{2}$ et $G_{3}$ dans la seconde population ( $p<0,01$ dans les deux cas) : il y a un excès d'hétérozygotes et un défaut d'homozygotes $A A$ au locus $G_{2}$, un défaut d'hérozygotes et un excès des deux génotypes homozygotes au locus $G_{3}$. On ne peut pour l'instant en proposer d'interprétation.

Quant à la relation de linkage entre les loci Ov et $\mathrm{G}_{3}$ (BUVANENDRAn, 1964 ; BuVAnENDRAN \& Finney, 1967), elle est refiétée clairement par le tableau 4. Ce dernier montre que l'on rencontre habituellement l'allèle $\mathrm{Ov}^{\mathrm{B}}$ lié à $\mathrm{G}_{3}{ }^{\mathrm{A}}$, et inversement $G_{3}{ }^{B}$ à $O v^{A}$, la combinaison la plus fréquente étant toutefois $O v^{A} G_{3}{ }^{A}$. Par contre, la combinaison $\mathrm{OvB}-\mathrm{G}_{3} \mathrm{~B}$ n'apparaît pas. Il en était de même dans les données de Buvanendran (1964, 1967 b), dans celles de MANwell \& BaKer (1970) et dans celles de Stratil (1968 a) citées et discutées par Manwell et BaKer. Seul Croizier (1968) a observé quelques individus porteurs de cette combinaison. D’autre part, le taux de recombinaison estimé sur nos données est de 7 p. 100. Ce taux paraît supérieur à celui observé par Buvanendran \& FinNey (1967), STratil (1968) et MANWEll \& BAKER (1970), résultats discutés dans leur ensemble par les deux derniers auteurs. De même, Crolzier (1968) trouve moins de 1 p. 100 de recombinaison entre les deux loci en question. Rappelons que nos deux populations, issues à l'origine d'un croisement de plusieurs races, pouvaient contenir une diversité génétique plus grande que celle des auteurs antérieurs. On ne peut peut-être exclure que, de ce fait, des facteurs modificateurs du taux de recombinaison aient été introduits.

\section{Proportions mendéliennes}

Le tableau 3 fait apparaître des écarts très hautement significatifs aux proportions mendéliennes prévues dans certains croisements. Au locus Ov, on remarque un excès du génotype $\mathrm{AA}$ sur le génotype $\mathrm{AB}$ dans les croisements où ces deux génotypes sont attendus en proportion égale, et, d'autre part, un excès du génotype $A B$ sur le génotype $\mathrm{BB}$ dans les croisements de type $\mathrm{AB} \times \mathrm{BB}$. Le croisement entre deux 
parents $A B$ montre la même tendance. On a donc partout un excès de l'allèle $A$ par rapport à la prévision.

Pour $\mathrm{G}_{3}$, par contre, les croisements $\mathrm{AA} \times \mathrm{AB}$ ou $\mathrm{AB} \times \mathrm{BB}$ et réciproquement, donnent des proportions très voisines de la prévision, mais, dans la descendance des croisements $\mathbf{A B} \times \mathbf{A B}$, on trouve un défaut de $\mathrm{BB}$ et un excès de $\mathrm{AA}$.

Quant à $\mathrm{G}_{2}$, l'interprétation est difficile, car les croisements $\mathrm{AB} \times \mathrm{AB}$ donnent beaucoup moins d'enfants AA que prévu, mais ce déficit n'est pas observé dans les croisements $\mathrm{AA} \times \mathrm{AB}$, peut-être du fait de leur faible effectif. Inversement, il y a un net défaut du génotype $A B$ par rapport à $B B$ dans les croisements $A B \times B B$ et réciproques, mais cela est moins clair dans le croisement $\mathrm{AB} \times \mathrm{AB}$. Globalement, ceci suggère que l'allèle $B$ est obtenu plus souvent que prévu dans les descendances en ségrégation.

Enfin, au locus Tf, la seule divergence significative par rapport aux proportions attendues est le manque d'enfants AA dans la descendance de parents tous deux AB.

Les écarts observés sont trop hautement significatifs pour être attribués à un hasard d'échantillonnage. Leur interprétation est d'autant plus difficile que l'identification des génotypes n'est faite que sur les poules en ponte, à l'âge tardif d'environ 9 mois. Dans les troupeaux et les années observées, la mortalité post-embryonnaire jusqu'à cet âge était relativement peu élevée, inférieure à 10 p. 100 dans l'ensemble et il paraît difficile de lui attribuer à elle seule les écarts les plus considérables. Quant à des différences de mortalité embryonnaire entre génotypes, le paragraphe suivant montre que, d'après du moins les comparaisons qu'il était possible de faire, elles ne sont significatives que dans un cas. On pourrait encore penser à une ponte inférieure des génotypes d'effectifs trouvés déficitaires lors du prélèvement des œufs pour leur identification, mais l'absence de différences de production d'œufs entre tous les génotypes montrée par les tableaux 5 à 8 (à l'exception possible d'une comparaison au locus $G_{2}$ ) rend cette hypothèse peu plausible. D'autres recherches sont donc nécessaires pour expliquer les présentes observations, en particulier des comparaisons des taux d'éclosion suivant le génotype des parents en effectifs plus importants.

Des déviations, par rapport aux proportions mendéliennes prévues, ont été mentionnées par BAKER $(1968 \mathrm{~b})$ : défaut d'hétérozygotes aux loci $\mathrm{Tf}, \mathrm{G}_{2}$ et $\mathrm{G}_{3}$ obtenu dans un troupeau de Sussex par cet auteur; déficit du génotype Tf $^{A}$ Tf $^{B}$ observé par BuVANENDRAN (1967 b) ; excès du même génotype trouvé par CroIZIER (1967). Peut-être nos propres résultats peuvent-ils rejoindre ceux de ces auteurs en ce qui concerne les loci $\mathrm{Ov}$ et $\mathrm{G}_{2}$, mais pas pour les loci $\mathrm{G}_{3}$ et $\mathrm{Tf}$.

\section{Comparaison des performances}

Du point de vue de la croissance pondérale, de la ponte et des caractéristiques des œufs, les tableaux 5 à 8 font apparaître très peu de différences significatives entre génotypes aux 4 loci étudiés : au locus $\mathrm{Ov}$, un pourcentage d’œufs mous ou sans coquille légèrement plus faible pour le génotype $B B$ que pour le génotype $A B$ ( $p<0,01$ ), au locus $\mathrm{G}_{2}$, le nombre d'œufs est plus élevé ( $\mathrm{p}<0,05$ ) pour AA que pour $A B ; G_{3}$ montre, seulement chez les poules de taille normale (Dw), un peu moins d'œufs cassés pour le génotype $\mathrm{AA}$ comparé à $\mathrm{AB}(\mathrm{p}<0,05)$, un léger avantage 
de AA sur $B B(p<0,05)$ pour le poids à 8 semaines, et de $B B$ sur $A B$ pour le poids à 8 semaines et le poids moyen des œufs $(p<0,05)$, mais les comparaisons correspondantes chez les poules dw n'indiquent pas de différence significative. Enfin, au locus Tf, le génotype $\mathrm{BB}$ comparé à $\mathrm{AB}$ a un poids corporel adulte et un poids moyen d'œufs inférieurs $(p<0,05)$ et une pigmentation des coquilles d'œufs un peu plus faible $(\mathrm{p}<0,01)$.

Le nombre limité de ces différences significatives, par rapport au nombre total des comparaisons, et le fait que la plupart ne sont significatives qu'au seuil de 5 p. 100 jette un doute sur leur réalité. Celles-ci devrait être confirmée par des données complémentaires. L'association des génotypes au locus Ov avec le pourcentage d'œufs mous et celle du locus $\mathrm{Tf}$ avec la coloration des coquilles paraissent pour l'instant les plus intéressantes à confirmer étant donné leur degré de signification. Notons en passant que la méthode des couples utilisée par nous diminue au maximum la variabilité génétique résiduelle, donc augmente le degré de sûreté des résultats. A notre connaissance, elle n'a pas été employée par les auteurs antérieurs.

Pour le taux d'éclosion, le tableau 9 ne montre qu'une différence significative consistant en une infériorité comprise entre 4 et $5 \mathrm{p}$. 100 pour les mères de génotype $\mathrm{BB}$ vis-à-vis de celles de génotypes $A B$ ou $A A$ au locus $G_{3}$, le seuil de signification atteint étant respectivement 5 p. 100 et 1 p. 100 suivant que ce taux est rapporté aux aufs incubés ou aux œufs fécondés. Ce seul résultat ne paraît pas de nature à expliquer les écarts aux proportions mendéliennes observées au même locus et discutées plus haut. Par contre, aux loci $\mathrm{Ov}$ et $\mathrm{G}_{2}$ où l'on note respectivement dans les ségrégations mendéliennes un excès et un défaut de l'allèle $A$, on peut remarquer, quoique les différences ne soient pas significatives, que le taux d'éclosion, respectivement, des mères $\mathrm{Ov}^{\mathrm{B}} \mathrm{Ov} \mathrm{B}$ comparées à celles des autres génotypes au locus $\mathrm{Ov}$, et des mères $\mathrm{G}_{2}{ }^{A} \mathrm{G}_{2}{ }^{\mathrm{A}}$ comparées à celles des autres génotypes au locus $\mathrm{G}_{2}$, est dans l'ensemble un peu inférieur. D'autre part, dans nos tests relatifs aux taux d'éclosion, nous avons dû nous limiter à la seule comparaison entre mères de certains génotypes. Cependant, le tableau 10 ne suggère pas d'effet du génotype paternel ni d'une interaction entre génotypes paternel et maternel sur l'éclosion. Notons également, toutefois, que dans l'ensemble le taux d'éclosion est relativement médiocre dans nos populations, pour des raisons que nous n'avons pu analyser (absence de sélection sur ce caractère? Présence au départ de races à taux d'éclosion suboptimal comme la Wyandotte... ?). Cela peut contribuer à masquer des différences dues ou associées à des variants des protéines de l'œuf, à moins que ces différences ne soient très importantes. Au total, il n'est pas évident, sans que cela soit non plus entièrement exclu, que certaines différences de mortalité embryonnaire entre génotypes puissent rendre compte des proportions mendéliennes anormales observées.

L'absence ou le caractère douteux des différences observées entre génotypes aux 4 loci étudiés pour les principaux caractères zootechniques dans notre étude englobant plusieurs années, semblent en accord avec les résultats publiés par plusieurs auteurs; par exemple Buvanendran (1967 b). Nous ne retrouvons pas dans le présent travail certaines différences obtenues par cet auteur d'une façon d'ailleurs non constante, non plus que celles suggérées par les résultats de Croizier (1969). Quant aux taux d'éclosion, nos résultats paraissent différer de ceux, par exemple, de MorTon et al. (1965) ou de BuVANENDRAN (1967 a). De même, nos populations n'extériorisent pas l'association importante et nette trouvée dans la race Fayoumi entre le génotype au locus $G_{2}$ et le poids moyen de l'œuf par OBEIDAH et al. (1977). Il se confirme 
donc que l'on ne peut généraliser les associations trouvées dans une population et dans des conditions particulières entre les génotypes aux quatre loci envisagées ici et des caractères de production. Sur un point, par contre, concernant la possibilité discutée par MANWELl et BAKer (1970) d'une sélection exercée contre certains génotypes aux deux loci liés $O v-G_{3}$, nos résultats paraissent concorder avec ceux présentés et discutés par ces auteurs.

Reçu pour publication en janvier 1982.

\section{Remerciements}

Nous remercions le $\mathrm{D}^{\mathrm{r}}$ C.M. Ann BAKER (Department of Zoology, University of Adelaide, Australie) de ses remarques intéressantes et constructives sur ce manuscrit, ainsi que le $\mathrm{D}^{r}$ Chevalet (Laboratoire de Génétique cellulaire, Centre de Recherches I.N.R.A., Toulouse-Auzeville).

\section{Summary \\ Frequencies and comparison of performance traits for genotypes at 4 loci controlling egg white proteins in the fowl}

In two experimental populations of domestic fowl, from 1973 to 1980 , the genotype of laying hens was identified for the 4 loci $O v, G_{m}, G_{3}$ and $T f$, responsible for genetic variants of egg white proteins detected by electrophoresis. Two alleles, A and B, were present in both populations at loci $G_{2}$ and $G_{3}$, and in one population at loci Ov and Tf. A high frequency is observed for the $A$ allele at loci $O v$ and $G_{3}$ and for the $B$ allele at loci $G_{2}$ and Tf, in accordance with observations of various authors. The linkage between $\mathrm{Ov}$ and $\mathrm{G}_{3}$, previously described, shows in our observations a recombination frequency of about 7 p. 100 .

The comparison of performance traits for body weight, egg number and egg traits, between genotypes at each locus on pairs of full- or half sisters shows, on the whole, only few significant differences, which should be confirmed on more data. For hatching percentage, the only statistical comparison which was possible on sufficient numbers was between dams of different genotypes mated to the same sires. It displayed one significant difference, related to $\mathrm{G}_{3}$ locus. On the other hand, the observation of mendelian proportions among progenies for each mating type at each locus showed in several cases highly significant deviations from expected proportions. An explanation of these deviations by differences of embryonic or post-embryonic mortality between certain genotypes is not obvious from our data.

\section{Références bibliographiques}

BAKER C.M.A., 1968 a. The proteins of egg white. In : "Egg quality. A study of the hen's egg », T.C. CARTER ed., pp. 67-108. Oliver \& Boyd, Edinburgh.

BAKER C.M.A., $1968 \mathrm{~b}$. Molecular genetics of avian proteins. IX - Interspecific and intraspecific variation of egg white protein of the genus Gallus. Genetics, 58, 211-226.

Baker C.M.A., Manwell C., 1962. Molecular genetics of avian proteins. I - The egg white proteins of the domestic fowl. Brit. Poult. Sci., 3, 161-174.

Baker C.M.A., Croizier G., Stratil A., Manwell C., 1970. Identity and nomenclature of some protein polymorphisms of chicken eggs and sera. Adv. Genet., 15, 147-174. 
Buvanendran V., 1964. Evidence of linkage between two egg albumen loci in the domestic fowl. Genetic Res., 5, 330-332.

BuVANEndRan V., 1967 a. A search for an association between maternal egg white protein polymorphisms and embryonic mortality in the domestic fowl. Brit. Poult. Sci., 8, 1-7.

BuVANENDRAN V., 1967 b. Egg-white polymorphisms and economic characters in the domestic fowl. Brit. Poul. Sci., 8, 119-129.

BuVAnendRan V., FinNey D.J., 1967. Linkage relationships of egg albumen loci in the domestic fowl. Brit. Poult. Sci., 8, 9-13.

Croizier G., 1966. Polymorphismes biochimiques de la poule domestique. I - Analyse génétique des protéines du blanc d'œuf chez des poules de races françaises et étrangères. Ann. Biol. anim. Biochim. Biophys., 6, 379-388.

Croizier G., 1967. Polymorphismes biochimiques de la poule domestique. II - Contrôle génétique du polymorphisme de la transferrine. Ann. Biol. anim. Biochim. Biophys., 7, 173-182.

Croizier G., 1969. Etude du polymorphisme des protéines de l'albumen chez Gallus Gallus L. Thèse, Paris.

GAHNE B., 1966. Studies on the inheritance of electrophoretic forms of transferrins, albumins, prealbumins and plasma esterases of horses. Genetics, 53, 681-694.

GilberT A.B., 1971. Egg albumen and its formation. In : «Physiology and Biochemistry or the domestic fowl ». Bell D.J. \& Freeman B.M. Editors, vol, III, pp. 1291-1329, Academic Press, London.

LusH I.E., 1961. Genetic polymorphisms in the egg albumen of the domestic fowl. Nature, 189, 981-984.

Manwell C., Baker C.M.A., 1970. Molecular biology and the origin of species : heterosis, protein, polymorphism and animal breeding. Sidgwick \& Jackson, London.

MéRAT P., 1967. Contribution à l'étude de la «valeur sélective 》 associée à quelques gènes chez la poule domestique. I - Différences quantitatives liées au génotype individuel. Ann. Biol., anim. Biochim. Biophys., 7, 75-104.

Merat P., 1969. Etude d'un gène de nanisme lié au sexe chez la poule. I - Description sommaire et periormances. Ann. Génét. Sél. anim., 1, 19-26.

Merat P., 1970. Mendelian genetics and selection for quantitative traits in poultry results and perspectives. World's Poult. Sci. J., 26, 571-586.

Morton J.R., Gilmour D.G., McDermid E.M., OGden A.L., 1965. Association of blood group and protein polymorphisms with embryonic mortality in the chicken. Genetics, 51, 997-1007.

Obeidah A., Merat P., Durand L., 1977. Polymorphism of egg white proteins, egg weight and component weight in the Fayoumi hen. Ann. Génét. Sél. anim., 9, 301-306.

Ogden A.L., Morton J.R., Gilmour D.G., McDermid E.M., 1962. Inherited variants in the transferrin and conalbumins of the chicken. Nature, 195, 1026-1028.

Stratil A., 1968. Ph.D. Thesis cited by Manwell \& Baker, 1970.

Stratil A., 1970. Genetic polymorphisms of proteins in different breeds and populations of chicken. Anim. Bd Gps Biochem. Genet., 1, 117-122. 